\title{
SEDA EN EPISIOTOMIAS Y DESGARROS. PERINEORRAFIA MODIFICADA
}

\author{
Dr. Alberto Zabaleta Lombana - Dr. Jorge Milanés P. \\ Departamento de Obstetricia y Ginecología - Facultad de Medicina - Cartagena \\ VI CONGRESO COLOMBIANO DE OBSTETRICIA Y GINECOLOGIA
}

En la práctica obstétrica ha llegado a ser la episiotomía un procedimiento necesario tan sencillo; $y$ su técnica de reparación tan simple, que escasamente habría algo que modificar en su rutina. Sin embargo, frecuentemente el dolor a nivel de los puntos de sutura demerita ante la paciente su importancia. Esta molestia atribuída al edema de los tejidos en el período expulsivo y a la contusión hemorrágica durante la práctica de maniobras instrumentales (1) ha sido objeto de variados tratamientos; procurando la reabsorción del edema mediante el empleo de lámparas de calor, anestesia tópica, soluciones inyectables locales, etc. Sherman y Ellison experimentaron una droga enzimática por vía oral, no encontrando mejores resultados que los obtenidos por el uso de un placebo. (2)

En parte se evitaría el edema prefiriendo la episiotomía media y utilizando la menor cantidad posible de un material de sutura delgado 30 pulgadas de catgut crómico 000, según Barter y col. (1)

Así, justificamos nosotros la utilidad de la seda mercerizada blanca № $80 ; y$ el tratamiento que prodiga- mos a la herida perineal, motivo de este trabajo.

Prácticamente hemos abandonado la episiotomía medio-lateral en favor de la mediana, más fácil de atender y de mejores resultados estéticos. Se le señala como desventaja la posibilidad de complicarse con desgarros que lesionen el recto. En sí, tal eventualidad no representa mayores inconvenientes; ya que es fácilmente subsanable con una sutura adecuada. Por lo demás, algunos autores propician el corte total del periné interesando deliberadamente el recto; o bien lo inciden cuando la integridad del mismo se ve amenazada por el desgarro (4-5).

\section{MATERIAL Y METODO}

Presentamos en esta experiencia 466 casos de episiotomía -en su inmensa mayoría mediana- y desgarros perineales en pacientes obstétricas de la Clínica Universitaria "Rafael Calvo C." de Cartagena. Fueron atendidas de la siguiente manera: anestesia local del periné con solución de Xilocaína al 1-2\%; y como elemento de sutura la seda blanca mercerizada $N$ ㅇ 80 que se esteriliza en cortas hebras sobre pequeños ca- 
rretes. Acerca de este material tenemos amplia experiencia, pues durante muchos años hemos venido suturando con él las laparotomías no infectadas y en la operación cesárea el útero y demás planos de incisión. (3) Los tejidos en la perineorrafia se adosan mediante puntos simples separados. Los cabos se cortan a nivel del nudo evitando sobrantes inoficiosos que al desaparecer la inhibición y el edema del parto, reaparezcan entre los labios de la herida entorpeciendo la cicatrización.

Introducimos una pequeña innovación al método corriente: No suturamos la mucosa vaginal ni los bordes de la piel. Los puntos a nivel de estos planos se anudan algunos milímetros por debajo de los mismos. Esto es suficiente para mantener en contacto las superficies seccionadas; a lo que contribuye eficazmente el cierre de los muslos. Sobre el cuerpo perineal los puntos separados se aplican según los requerimientos de la herida.

En cuanto a los desgarros de tercer grado (D. III) que comprometen el esfínter del ano, son separados de la manera acostumbrada, tomando los extremos del esfínter con seda. Los de cuarto grado o completos (D. IV) que interesan la mucosa rectal abriendo esta cavidad, son suturados con dos planos a puntos separados sobre la fascia perirectal, poniendo especial cuidado para evitar puntos perforantes. (6-7) Los restantes tiempos, de la manera anotada para los D. III y las episiorrafias.

Durante el puerperio no se hizo antibióticoterapia sino cuando ello se consideró conveniente. Se recomendó el uso de toallas sanitarias, lo que no fue posible para muchas. Por el hecho de la episiotomía no se demoró más de 24 horas la estadía hospita- laria, la mayoría de las veces por solicitud de la paciente, alegando sentirse bien y ser indispensable su presencia en el hogar. De otra parte, el gran volumen de puérperas y la escasa capacidad de la Clínica obligan a acortar al mínimo el internamiento en los casos normales.

Las pacientes que sufrieron desgarro completo (D. IV) se les mantuvo durante 72 horas y recibieron tratamiento con antibiótico.

Las heridas eran observadas cada día durante la permanencia hospitalaria; y posteriormente controladas a los 8-30 y 60 días. Para estos términos se investigaba las condiciones estéticas de la cicatriz, edema, hematomas, infección y dolor $u$ otro síntoma cualquiera que pudiera ser atribuído al material o método de sutura. En las dos últimas visitas se examinaba, además, por tacto rectal, la tonicidad del esfínter y elevadores del ano. No manifestaron dispareumia alguna pacientes que para entonces habían tenido relaciones sexuales. A todas se les solicitaba nos consultaran en caso de tal molestia.

Las pacientes no fueron seleccionadas. Las hubo con marcada anemia desnutridas; y con pre-eclampsia, eclampsia. Tres tenían bartolinitis crónica. Las suturas fueron tomadas por los autores del presente trabajo, los internos del servicio y alumnos del sexto año de medicina.

La edad de las pacientes estuvo comprendida entre los 14 y los 38 años con una mayor frecuencia por debajo de los 20 años. Se anotó la gravidez en 401 casos; de los cuales $289(72 \%)$ fueron primigrávidas, 61 (15.2\%) secundigrávidas, 25 tercigrávidas y las restantes grandes multíparas. 
Hemos calificado los resultados de la siguiente manera: Excelente cicatrización anatómica y funcionalmente correcta. No hubo necesidad de retirar puntos; ni manifestación alguna de molestia, dolor, edema o infección.

Bueno: La cicatrización es correcta. Puede haber ligero edema, dolor moderado o peso cuando la paciente se pone en pie o sentada, referido al periné. Hay necesidad de retirar algún hilo que aflora entre los bordes de la herida o en pequeñas vesículas de contenido seroso.

Regular: Defecto de adosamiento, defecto de técnica, de los planos de mucosa vaginal o piel. Necesidad de retirar puntos de hilo, a veces de minúsculas vesículas a nivel de los mismos.

Malo: Cuando la deficiente técnica cie sutura afronta mal los tejidos retardando la cicatrización. Puede haber dehiscencia, marcado edema o infección y consiquiente curación por segunda intención.

\section{MORBILIDAD}

Ningún caso hizo necesaria su permanencia más allá del tiempo indicado anteriormente, o su posterior internamiento. Siete pacientes presentaron durante cl primer control ligero edema de los bordes de la herida. De 251 señoras que concurrieron a las visitas de control, solamente 21 $(8.3 \%)$ acusaron dolor moderado referido al periné al sentarse, levantarse $\circ$ al defecar, molestias éstas que desaparecieron para el siguiente control al retirar uno que otro punto de seda. Algunas refirieron ardor durante la micción, por defecto de coaptación de los bordes.

En estos casos, los extremos del hilo fueron cortados muy largos, la mayoría de las veces; o bien los puntos de sutura colocados muy superficialmente. En algunas pacientes con dos controles, se retiró en el primero algún punto, calificando el resultado como bueno; para considerarlo excelente en el segundo examen por la perfecta cicatrización.

En 26 casos (10\%) hubo necesidad de retirar puntos, así: 12 veces un hilo; 3 veces dos hilos; 7 veces tres hilos y 4 hilos en un caso.

Hemos reunido en 10 cuadros los datos estadísticos que integran la observación de las 466 pacientes estudiadas. En el Cuadro № 1 recopilamos los apartes referentes al tipo de lesión reparada, su frecuencia y porcentaje.

\section{CUADRO N: 1}

466 EPISIOTOMIAS Y DESGARROS. INCIDENCIA

\begin{tabular}{|c|c|c|}
\hline Lesiones & Casos & Porcentaje \\
\hline $\begin{array}{l}\text { Episiotomía Mediana } \\
\text { Episiotomía media c/Desg. }\end{array}$ & 347 & 74,5 \\
\hline $\begin{array}{l}\text { Incom. } \\
\text { Episiotomía media c/Desg. }\end{array}$ & 12 & 2,5 \\
\hline Comp. & 13 & 2,7 \\
\hline Episiotomía medio lateral & 10 & 2,1 \\
\hline Desgarro II & 75 & 16,2 \\
\hline Desgarro 111 & 6 & 1,2 \\
\hline Degarro IV & 2 & \\
\hline TOTAL & $466 p$ & cientes \\
\hline
\end{tabular}

La episiotomía mediana ocupa el primer lugar entre las lesiones maternas $(74.5 \%)$ pues se practica rutinariamente. Las pocas medio laterales anotadas se hicieron con fines de docencia. Las episiotomías medias complicadas con D. III y D. IV, fueron debidas en su mayoría a aplicaciones de fórceps. El segundo lugar lo ocupa el desgarro de segundo gra. do con el $16.2 \%$. 


\section{CUADRO NN 2}

466 PACIENTES. CONTROLES Y PORCENTAJES

\begin{tabular}{ccc}
\hline No de controles & No de casos & Porcentajes \\
\hline \multicolumn{4}{c}{1} & 175 & 37,5 \\
2 & 57 & 12,3 \\
3 & 19 & 4 \\
Sub total & 251 & 53,8 \\
0 & 215 & 46,1 \\
TOTAL & 466 pacientes \\
\hline
\end{tabular}

Doscientos cincuenta y una pacientes -53.8 - del total asistieron a uno, dos y tres controles; por el contrario, $215(46.1 \%)$ nunca concu-

CUADRO N: 3

215 PACIENTES CON O CONTROL. LESIONES

\begin{tabular}{llrc}
\hline Lesiones & & Casos & Porcentajes \\
\hline Episiotomía Media & & 149 & 69,3 \\
Episiotomía media c/Desg. & & \\
$\quad$ Incom. & & 8 & 3,7 \\
Episiotomía media c/Desg. & 5 & 2,3 \\
$\quad$ Compl. & & 5 & 2,3 \\
Episiotomía medio lateral & 45 & 20,9 \\
Desgarro II & & 2 & \\
Desgarro III & & 1 & \\
Desgarro IV & & 215 pacientes \\
\cline { 2 - 3 } TOTAL & &
\end{tabular}

rrieron. Las particularidades de estos últimos las agrupamos en el Cuadro № 3.

CUADRO N: 4

357 EPISIOTOMIAS. - RESULTADOS

\begin{tabular}{lrrrrr}
\hline Control & Excelente & Bueno & Regular & Malo & Total \\
\hline 1 & 71 & 47 & 19 & 5 & 142 \\
2 & 25 & 16 & 4 & 1 & 46 \\
3 & 9 & 6 & 23 & 6 & 203 \\
$\begin{array}{l}\text { Subtotal } \\
\text { O control }\end{array}$ & 105 & 69 & & & 154 \\
$\begin{array}{l}\% \text { sobre 203 } \\
\text { controladas }\end{array}$ & 51,7 & 34 & 11,3 & 2,9 & \\
\hline
\end{tabular}

Recogemos en el Cuadro Ni 4 los control 154 pacientes (43.1\%). Los 347 casos de episiotomía mediana y restantes $2 u$ u $(56.9 \%)$ con uno, dos los 10 de episiotomía media lateral, y tres visitas, se clasificaron excelensobre los cuales dejaron de asistir a tes $105(51.7 \%$ ) y buenos $6934 \%$.

\section{CUADRO N: 5}

25 EPISIOTOMIAS COMPLICADAS CON DESGARROS:

12 INCOMPLETOS - 13 COMPLETOS - RESULTADOS

\begin{tabular}{ccccrc}
\hline Control & Excelente & Bueno & Regular & Malo & Total \\
\hline 1 & 5 & 2 & 1 & - & 8 \\
2 & 1 & 1 & - & - & 2 \\
3 & 1 & 3 & 1 & 12 \\
Subtotal & 7 & & & 13 \\
O control & & & & 12 \\
\hline
\end{tabular}


De las 25 pacientes del Cuadro № 5 solamente acudieron a control 12 , de las cuales 7 se consideraron excelentes y 3 buenas.

Sumados los datos de ambos cua- dros encontramos que sobre $215 \mathrm{pa}$ cientes de episiotomías simples y complicadas con desgarros con uno, dos y tres controles, 184 (112 excelentes y 72 buenas) curaron su lesión en forma satisfactoria (85.5\%).

\section{CUADRO N: 6}

84 DESGARROS PERINEALES: 76 D. II - 6 D. III - 2 D. IV RESULTADOS

\begin{tabular}{cccccc}
\hline Control & Excelente & Bueno & Regular & Malo & Total \\
\hline 1 & 11 & 12 & 3 & - & 26 \\
2 & 2 & 2 & 3 & - & 7 \\
3 & 3 & 14 & 6 & - & 3 \\
Subtotal & 16 & & & 36 \\
O control & & & & 48 \\
\hline
\end{tabular}

De estos 84 pacientes con desgarros, 36 (42.8\%) volvieron a control; siendo los resultados excelentes y buenos en número de 30 para un $83.3 \%$.

\section{CUADRO N: 7}

251 PACIENTES CONTROLADOS RESULTADOS

\begin{tabular}{|c|c|c|c|c|c|c|c|}
\hline \multirow[b]{2}{*}{ Resultados } & \multicolumn{4}{|c|}{ Episiotomías } & \multirow[b]{2}{*}{ Desgarros } & \multirow[b]{2}{*}{ Totales } & \multirow[b]{2}{*}{$\%$} \\
\hline & $\operatorname{Sin}$ & desgarro & Con & desgarro & & & \\
\hline Excelente & & 105 & & 7 & 16 & 128 & 50.9 \\
\hline Bueno & & 69 & & 3 & 14 & 86 & 34.3 \\
\hline Regular & & 23 & & 1 & 6 & 30 & 11.9 \\
\hline Malo & & 6 & & 1 & 0 & 7 & 2.7 \\
\hline TOTAL & & 203 & & 12 & 36 & 251 & \\
\hline
\end{tabular}

Finalmente, el Cuadro № 7 recoge globalmente los resultados parciales sobre 251 pacientes con uno, dos y tres controles. Los excelentes y buenos totalizan 214 casos para un porcentaje de $85.2 \%$; lo que indica los beneficiosos resultados que se obtienen con la técnica y material de sutura empleados por nosotros en la reparación de las lesiones obstétricas del periné.

Del gran total de 466 pacientes objeto de este trabajo, 40 fueron controladas a su regreso a la Clínica para un nuevo parto; uno o dos años más tarde (1964-1965). Sus antecedentes en relación con este estudio -número de controles y tipo de lesión reparada- aparecen en el Cuadro № 8.

De estas 40 pacientes, 17 no asistieron a control alguno en su embarazo anterior cuando les fue reparada su lesión perineal. Las restantes $23(57.5 \%)$ atendieron las visitas, siendo calificadas 20 como excelentes y buenas y 3 como regular. 
CUADRO N: 8

Detalles de 40 PACIENTES EN UN NUEVo EMBARAzO

\begin{tabular}{ccccccrr}
\hline Control & Excelente & Bueno & Regular & Total & Tipo de lesión & Casos \\
\hline 1 & 6 & 5 & 1 & 12 & Episiotomía Media & 32 \\
2 & 3 & 3 & 2 & 8 & Episiotomía mas D. IV & 2 \\
3 & 1 & 2 & - & 3 & Desgarro II & 5 \\
Subtotal & 10 & 10 & 3 & 23 & Desgarro III & 1 \\
0 control & & & & 17 & TOTAL & 40 \\
\hline
\end{tabular}

\section{CUADRO № 9}

40 PACIENTES - DETALLES DEL PARTO

\begin{tabular}{lrllllr}
\hline Tipo de parto & Casos & Horas de hospital & Casos & Lesiones maternas & Casos \\
\hline Espontáneo & 35 & Expulsión & 10 & Episiot. media & 7 \\
Fórceps & 2 & Menos de 12 & 24 & Episiot. mas D. III & 1 \\
Moriceau & 1 & 20 & 2 & Desgarro II & 5 \\
Abortos & 2 & Parto en casa & 2 & Desgarro III & 3 \\
TOTAL & 40 & & & Ninguna lesión & 24 \\
\hline
\end{tabular}

En el Cuadro № 9 detallamos la modalidad del parto motivo del nuevo control de estas 40 pacientes. Dos de ellas regresaron por aborto.

En la columna "horas de hospital" anotamos el tiempo transcurrido desde la llegada de la paciente a la Clínica hasta la verificación del parto. Las dos parturientas con 20 horas tuvieron los fetos de mayor peso: 4.250 y 4.050 gramos respectivamente, uno de ellos en presentación de nalgas. $24(60 \%)$ no sufrieron lesión perineal alguna. Ninguna de estas pacientes acusó dispareumia; no obstante que en dos de ellas se encontró sendos hilos intraperineales.

\section{CUADRO No 10}

\section{PESO FETAL EN GRAMOS}

\begin{tabular}{|c|c|c|}
\hline \multicolumn{2}{|c|}{1.000 gramos } & 1 \\
\hline 2.500 & 3.000 & 19 \\
\hline 3.090 a & 4.250 & 19 \\
\hline Abortos & & 2 \\
\hline TOTAL & & 41 \\
\hline
\end{tabular}

Finalmente en el Cuadro № 10 detallamos los pesos de los fetos en gramos. Hubo un parto gemelar; y se consignan dos abortos. Los fetos todos nacieron vivos.

\section{RESUMEN}

Presentamos 466 casos de episiotomías medianas, algunas medio laterales y desgarros de la región perineal en pacientes obstétricas. Fueron reparados con seda blanca mercerizada número 80 , mediante una modificación de la técnica utilizada comunmente, así: puntos sencillos separados; dejando sin suturar la mucosa vaginal y la piel del periné, cuyos bordes son simplemente adosados por los puntos profundos.

Asistieron a uno, dos y tres controles, cada 8-30 y 60 días, 251 (53.8\%) pacientes. Sus resultados altamente favorables al procedimiento son consignados en 6 cuadros parciales y 1 general; dando un porcentaje final de 85.2 (214 pacientes) para los casos excelentes y buenos. 
De estas 466 pacientes regresaron para un nuevo parto, uno o dos años más tarde, 40 señoras cuyos detalles son anotados en los cuadros 8, 9 y 10. En este trabajo intervinieron los autores del mismo, los internos de la unidad obstétrica y alumnos del sexto año de medicina.

\section{CONCLUSIONES}

1) La seda es un material aceptable como elemento de sutura en las episiotomías y desgarros, aun en los de cuarto grado del periné.

2) Nuestra técnica muestra que es inoficiosa la sutura de la mucosa vaginal y de la piel del periné; ya que sin ella curan satisfactoriamente.

3 ) Se observa mínima incidencia de morbilidad y molestias dolorosas en la región perineal.

4) El retiro de algunos puntos que por su colocación superficial afloran entre los bordes de la herida o forman pequeñas vesículas, da lugar a una buena cicatrización.

5) Esta cicatrización cursa sin fibrosis del periné ni da posteriormente lugar a dispareumia.
6) Se observa una pronta y normal integridad de la mucosa vaginal y piel del periné.

7) Los resultados son tanto más satisfactorios cuanto mayor es la experiencia del operador.

\section{BIBLIOGRAFIA}

1) BARTER R. H., PARKS J. TYNDAL CH: Median episiotomies and complete perineal lacerations. Amer. J. Obst. Gynec. Vol. 80. No 4,1960

2) SHERMAN E., ELLISON R. S.: Subjective evaluation of an enzime preparation in episiotomy pain. Amer. J. Obst. Gynec. Vol. 82 1961.

3) ZABALETA LOMBANA A., MILANES J.: Sutura con seda en la operación cesárea. Rev Col. Obst.-Ginec. Vol. XIV. Trib. Med. Vol. 841963

4) BRANTLEY J. T., BURWELL J. C.: A study of fourth degree perineal lacerations and their sequelae. Amer. J. Obst. Gynec. Vol. 801960.

5) FERNANDEZ BASTIDAS M. A.: Técnica original de perineorrafia post episiotomía. Rev. Col. Obst. Ginec. Vol. VI. Nov. Dic. 1954.

6) EASTMAN N. J.: Obstetricia de Williams. Pág. 438. $3^{\text {ạ }}$ ed. española. Uteha. México, 1960 .

7) DEXEUS FONT S.: Tratado de Obstetricia. Pág. 1041. 1: ed. Salvat Editores. Barcelona-Madrid 1949. 